\title{
REFLEXÕES SOBRE O TRAUMA CARDIOVASCULAR CIVIL A PARTIR DE UM ESTUDO PROSPECTIVO DE 1000 CASOS ATENDIDOS EM UM CENTRO DE TRAUMA DE NÍVEL I
}

\author{
REFLECTIONS ABOUT CIVILIAN CARDIOVASCULAR TRAUMA ADMITTED TO A \\ LEVEL 1 TRAUMA CENTER: A PROSPECTIVE STUDY FROM 1000 CASES
}

\author{
Ricardo Costa-Val'; ; Sérgio Figueiredo Campos-Christo²; \\ Wilson Luiz Abrantes, TCBC-MG3; Marcelo Barroca Campos-Christo, ECBC-MG ${ }^{4}$; \\ Maria Cristina Marques ${ }^{5}$; Eduardo Vergara Miguel $^{6}$
}

\begin{abstract}
RESUMO: Objetivo: Analisar os dados referentes a uma grande série de casos de traumas cardiovasculares exclusivamente civil operados em um único centro de trauma brasileiro. Método: Trata-se de um estudo de coorte, prospectivo, descritivo e analítico registrados entre os anos de 1998 - 2005. Resultados: No período foram operados 1000 casos que acometeram principalmente homens jovens devido a armas de fogo, armas brancas/vidros e trauma contuso e cuja topografia das lesões se deu na seguinte ordem: abdominais, cervicais, torácicas e extremidades. As três síndromes mais comuns a admissão foram: hemorrágica, isquêmica e hemorrágica/isquêmica. No entanto, $34.6 \%$ dos pacientes estavam em choque hipovolêmico grave e em $85 \%$ da casuística havia lesões não cardiovasculares associadas. A maioria dos pacientes foi submetida a tratamento cirúrgico sem propedêutica específica, mas $14 \%$ destes foram reoperados devido à síndrome compartimental, trombose aguda e/ou hemorragia grave. A taxa de amputação foi de $5.5 \%$ e da mortalidade de $7.5 \%$, estando correlacionada com choque hipovolêmico grave ou síndrome da resposta inflamatória sistêmica. Lesões CCV isoladas ocorreram em $15 \%$ dos casos com taxa de letalidade global de $41 \%$, sendo $22 \%$ venosa, $47 \%$ arterial e $81 \%$ cardíaca, proporcionando diferença significativa entre lesões cardíacas versus arteriais e venosas associadas $(p=0,01$; odds ratio de 7.37) e lesões arteriais versus venosas $(\mathrm{p}=0,01$; odds ratio de 3.17). Conclusão: Esta grande série de casos demonstrou ser o homem jovem o mais acometido devido principalmente à violência interpessoal e os acidentes automobilísticos, que envolvem na maioria das vezes as extremidades, associadas com lesões em outros órgãos ou sistemas. Os fatores preditivos de mau prognóstico foram choque hipovolêmico, lesão de grandes vasos arteriais e lesão cardíaca (Rev. Col. Bras. Cir. 2008; 35(3): 162-167).
\end{abstract}

Descritores: Sistema cardiovascular/lesões; Ferimentos e lesões; Isquemia; Reperfusão; Medicina baseada em evidências; Epidemiologia; Procedimentos cirúrgicos vasculares.

\section{INTRODUÇÃO}

Relatos sobre trauma são tão antigos quanto à própria história do Homem ${ }^{1,2}$. No entanto, o trauma tornou-se um dos maiores desafios para o sistema assistencial de saúde de todos os países do mundo, sendo o trauma vascular componente importante deste problema ${ }^{3-14}$. Apesar disso, a maioria das grandes séries sobre trauma cardiovascular é baseada em conflitos militares ${ }^{1,2,9,15-17}$, pois estudos epidemiológicos sobre trauma cardiovascular civil são difíceis de serem realizados e analisados ${ }^{18-20}$. Por outro lado, a epidemiologia clínica tem se revelado como poderosa ferramenta capaz de preencher lacunas científicas e proporcionar uma melhor compreensão dos fenômenos sociais e agravos à saúde existentes ${ }^{21-29}$. Atualmente, os estudos epidemiológicos observacionais, prospectivos baseados em grande série de casos são considerados Nível II de Evidência Científica ${ }^{23-25}$, o que se aplica a este trabalho por ser uma das maiores séries de traumas cardiovasculares de etiologia exclusivamente civil pertencentes a um único centro de trauma. Soma-se a este fato a importância e necessidade de conhecermos os dados regionais e nacionais a respeito do trauma cardiovascular, já que existem grande variações nos fatores etiológicos e nos resultados pós-operatórios dos mesmos em virtude das diferentes características das sociedades ao redor do mundo ${ }^{3,4,10-14,17-20,30}$.

O objetivo deste trabalho é analisar os dados referentes a uma grande série de casos de traumas cardiovasculares exclusivamente civis operados em um único centro de trauma brasileiro.

\footnotetext{
1. Titular da Sociedade Brasileira de Angiologia e Cirurgia Vascular (SBACV); Mestre e Doutor em Medicina Pela Universidade Federal de Minas Gerais - UFMG.

2. Doutor em Medicina pela UFMG.

3. Pioneiro da Cirurgia do Trauma em Minas Gerais.

4. Pioneiro da Cirurgia Cardiovascular do Trauma em Minas Gerais.

5. Enfermeira Coordenadora do Centro Cirúrgico do Hospital João XXIII e Gerente Administrativa da FHEMIG.

6. Coordenador do Serviço de Trauma Cardiovascular - Hospital João XXIII /FHEMIG.
}

Recebido em 16/11/2007

Aceito para publicação em 15/01/2008

Conflito de interesses: nenhum

Fonte de financiamento: nenhuma

Trabalho realizado no Hospital João XXIII/FHEMIG - Belo Horizonte/MG. 


\section{MÉTODOS}

Modelo de estudo e aspectos éticos: o registro de todos os casos de trauma cardiovascular no protocolo específico foi autorizado pela Diretoria do HPS/João XXIII e não há neste estudo a possibilidade de identificação dos pacientes analisados. De igual modo não existem atitudes por parte de nenhum dos autores que possa ser caracterizada como conflito de interesse.

Trata-se de um estudo de coorte, prospectivo, descritivo e analítico sobre casos registrados pelo Serviço de Trauma Cardiovascular do referido hospital, entre os anos de 1998 - 2005, utilizando-se o Programa SPSSÒ versão 12.0 e tendo sido estabelecida uma margem de erro de $5 \%$ ( $\mathrm{p} £ 0.05$ ) para rejeição da hipótese de nulidade em relação às análises comparativas realizadas. Utilizou-se o Teste de Kruskall-Wallis entre as análises estatísticas e as chances de morte (odds ratio) em relação ao local da lesão cardiovascular isolada. Foram utilizados para a análise crítica dos resultados dados da literatura nacional e da língua inglesa desde 1990 encontrados nas fontes de dados Medline, LILACS e SCIELO Brazil e cujas palavras-chaves foram trauma, trauma cardiovascular, isquemia, epidemiologia clínica.

Critérios de inclusão: pacientes portadores de lesões cardiovasculares de etiologia civil operados pela Cirurgia Cardiovascular e/ou Cirurgia do Trauma, registrados no protocolo específico.

Critérios de exclusão: foram excluídos os pacientes não registrados imediatamente após o ato operatório, pacientes admitidos "in extremis" e/ou recebendo massagem cardíaca externa devido à parada cardiorrespiratória ou lesões sabidamente ocorridas em soldados pertencentes às forças armadas brasileiras durante qualquer tipo de atividade militar.

Local do Estudo: Hospital João XXIII, classificado como Centro de Trauma Nível I, pertencente à Fundação Hospitalar do Estado de Minas Gerais - FHEMIG.

\section{Registro de Trauma Cardiovascular}

O registro de trauma do serviço supracitado foi criado em 1997 e pode ser subdividido em nove grandes grupos, a saber: Grupo 1: nome, idade, sexo, profissão e endereço; Grupo 2: dados referentes à história e a cinemática do trauma e as condutas tomadas durante a fase pré-hospitalar; Grupo 3: dados referentes as condições clínicas do paciente à admissão na sala de emergência; Grupo 4: dados referentes aos exames pré-operatórios realizados; Grupo 5: dados referentes a lesões não vasculares associadas e a outras cirurgias realizadas;

Grupo 6: descrição da lesão vascular. Grupo 7: descrição das técnicas cirúrgicas realizadas; Grupo 8: evolução pós-operatória até o momento da alta ou transferência hospitalar; Grupo 9: comentários adicionais e identificação da equipe cirúrgica.

\section{RESULTADOS}

Durante o período citado foram registrados $1000 \mathrm{ca}-$ sos de traumas cardiovasculares exclusivamente civis, ocorri- dos na sua grande maioria em homens $(88 \%)$ jovens (média das idades de 26.9 anos; idade mínima de dois anos e máxima de 84 anos) devido principalmente a lesões provocadas pelos diversos tipos de armas de fogo $(45.1 \%)$, armas brancas/vidros $(30.8 \%)$, traumas contusos $(23.2 \%)$ e iatrogênicos $(1.1 \%)$.

De acordo com a região anatômica acometida, se constatou que $6.1 \%$ das lesões ocorreram no abdome, $8.6 \%$ na região cervical, $10.3 \%$ no tórax e em $2 / 3$ dos casos, nas extremidades, sendo $36.5 \%$ nos membros superiores e $38.4 \%$ nos membros inferiores, sendo $63.5 \%$ arteriais, $35.4 \%$ venosas e $1.1 \%$ cardíacas. Em $28.1 \%$ dos pacientes havia tanto lesão arterial quanto venosa.

As principais síndromes clínicas apresentadas à admissão foram: hemorrágica (39.4\%); isquêmica (27\%); hemorrágica e isquêmica (11.6\%); tumoral (8.5\%); isquêmica e tumoral (4.7\%); hemorrágica e tumoral (3.8\%). Apenas $2.8 \%$ dos pacientes apresentavam manifestações clássicas da síndrome de tamponamento cardíaco e $2.2 \%$ de síndrome compartimental periférica. O choque hipovolêmico grave (Grau III ou IV) foi observado em $34.6 \%$ dos pacientes admitidos.

A associação dos traumas CCV com lesões em outros órgãos ou sistemas ocorreu em 850 (85\%) casos, sendo as associações mais prevalentes: fraturas $(29 \%)$; lesões nervosas $(24 \%)$, lesões de grandes partes moles $(23 \%)$, lesões dos órgãos toraco-abdominais (14\%). Em 10\% da amostra havia amputação periférica traumática parcial .

A maioria dos pacientes $(84.7 \%)$ foi submetida a tratamento cirúrgico imediato baseado apenas no exame físico com Doppler de ondas contínuas e/ou devido às lesões não cardiovasculares associadas. Os exames propedêuticos préoperatórios específicos mais utilizados foram: arteriografia $(56.9 \%)$; dúplex-scan (17.1\%); tomografia computadorizada contrastada (16.6\%) e ecocardiograma (9.4\%).

As artérias mais acometidas foram: braquial (15.3\%); femoral (15\%); poplítea (11\%); ulnar e/ou radial (6.3\%); tibial posterior (3.5\%); carótidas (3.4\%); axilar (3.1\%); subclávia (3\%); ilíacas $(2.2 \%)$ e aórtica $(0.7 \%)$.

As veias mais atingidas foram: femoral (9.6\%); ulnar e radial (7.1\%); poplítea (3.2\%); jugular interna (3\%); braquial (2\%); subclávia (1.4\%); veia cava inferior $(1 \%)$; axilar $(0.5 \%)$; renal $(0.3 \%)$ e outras menores tais como jugular externa, safena magna e safena parva, tibiais, cefálica e basílica (7.3\%).

O tratamento das lesões arteriais foi feito por meio de sutura primária, interposição e/ou by-pass (venoso e/ou sintético) em $91 \%$ dos casos, sendo que $14 \%$ destes tiveram que ser reoperados devido principalmente a síndrome compartimental periférica por fenômeno de isquemia/ reperfussão (23\%), isquemia crítica por trombose arterial aguda $(20 \%)$ e hemorragia com repercussões hemodinâmicas (13\%). A amputação primária foi indicada em 1.3\% dos pacientes. Entretanto, $4.2 \%$ dos pacientes foram submetidos à amputação secundária devido à isquemia irreversível e/ou infecção grave. Já as principais opções técnicas realizadas para as lesões venosas foram: ligadura venosa primária (57.6\%), sutura venosa $(26.3 \%)$, enxerto venoso $(7.3 \%)$, sutura com remendo $(5.5 \%)$ e anastomose termino-terminal (3.3\%). A heparinização sistêmica e/ou local foi utilizada em $51 \%$ das revascularizações arteriais, não sendo realizada naqueles pa- 
cientes que tinham contra-indicações devido a outras lesões associadas e/ou pelas condições clínicas per-operatórias.

A mortalidade global da série foi de $7.5 \%$ (75 pacientes), sendo as duas causas principais correlacionadas: choque hipovolêmico grave à admissão ou síndrome da resposta inflamatória sistêmica/falência de múltiplos órgãos.

As principais lesões cardiovasculares não isoladas que estiveram associadas ao óbito hospitalar foram: artéria femoral (21.4\%); cardíaca (12\%); artérias ilíacas (11.6\%); aorta, artéria subclávia e veia cava inferior ( $9.3 \%$ cada); artérias carótidas (6.7\%); veias ilíacas $(5.3 \%)$, vasos do pedículo pulmonar $(4.9 \%)$, mamária interna $(3.7 \%)$, vasos viscerais tronculares, tais como tronco celíaco, mesentérica superior e hepática $(2.6 \%)$ e artéria poplítea, veia subclávia e veia renal (1.3\% cada).

Ocorreram 150 (15\%) lesões cardiovasculares isoladas, definidas como apenas lesão arterial ou venosa ou cardíaca, distribuídas da seguinte maneira: $89(59.3 \%)$ arteriais, 50 (33.3\%) venosas e $11(7.4 \%)$ cardíacas com uma taxa de letalidade hospitalar de $41 \%$, sendo $22 \%$ devida à lesão venosa, $47 \%$ por lesão arterial e $81 \%$ por lesão cardíaca, conforme Tabela 1.

A análise comparativa entre as mortes por lesões cardiovasculares isoladas mostrou ter havido diferença significativa entre as lesões cardíacas em relação às lesões arteriais e venosas associadas $(\mathrm{p}=0,01)$ e entre as lesões arteriais em relação às lesões venosas $(\mathrm{p}=0,01)$ (Figura 1). Já a chance de

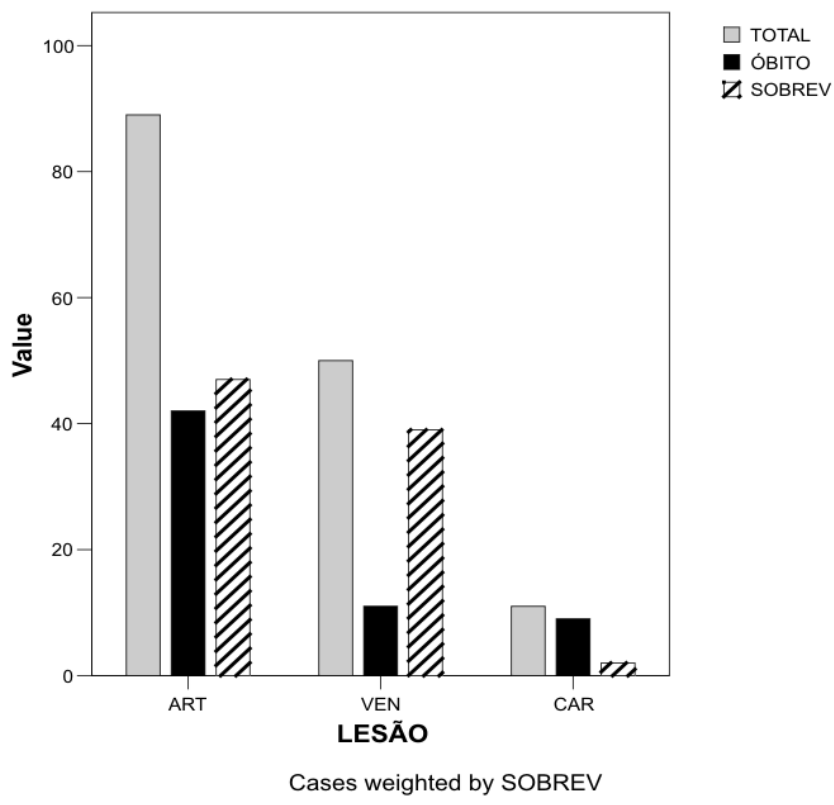

Figura 1 - Análise comparativa entre óbitos hospitalares por lesões cardiovasculares isoladas. morte (odds ratio) demonstrou ser de 7.37 para as lesões cardíacas em relação aos traumas arteriais e venosos associados e de 3.17 para as lesões arteriais em relação aos traumas venosos.

- Letalidade entre óbitos por lesão cardíaca versus lesões arteriais e venosas: $\mathrm{p}=0.01$ (Teste de Kruskall-Wallis).

- Letalidade entre óbitos por lesão arterial versus venosa: $\mathrm{p}=0.01$ (Teste de Kruskall-Wallis).

\section{DISCUSSÃO}

Sabe-se que uma das principais dificuldades em se aprimorar a assistência à saúde, seja ela individual ou coletiva, reside na incapacidade de se dominar as concepções teóricas, particularmente sobre fatores antropológicos, sociais e etiológicos relacionados às doenças e agravos à saúde de qualquer sociedade, se destacando neste contexto a epidemiologia clínica e as práticas assistências associadas às evidências científicas ${ }^{4,9,21-27,31,32}$.

Ao lidarmos com estudos sobre trauma vascular devemos obrigatoriamente pensar no pioneirismo de Norman Rich, destacando-se o clássico Vietnam Vascular Registry, apesar deste ser baseado em conflitos militares ${ }^{1,2}$.

Notório se faz mencionar que uma das grandes dificuldades em se realizar estudos epidemiológicos a respeito do trauma cardiovascular civil não está na falta de qualidade técnica-científica do profissional/pesquisador brasileiro, mas sim na omissão em se publicar trabalhos e estudos que, muitas vezes ficam engavetados ${ }^{26}$. No entanto, se nota uma crescente preocupação nacional sobre isso, demonstrando uma positiva mudança de comportamento ${ }^{5-14,18,19,33-37}$

A grande casuística registrada em um único centro de trauma brasileiro corrobora estudos multicêntricos realizados na América Latina que constataram que a maioria dos casos foram provenientes do Brasil ${ }^{28,29}$.

Dados da Organização Mundial de Saúde relatam mais de cinco milhões de óbitos em 2000 e estimam em 8,5 milhões de óbitos devido às conseqüências do Trauma em 2010 ao redor do mundo, fazendo do Trauma uma pandemia e um dos maiores desafios da Medicina Moderna ${ }^{3,4}$.

Por outro lado, sabe-se que o registro institucional dos Centros de Trauma, apesar das possíveis sub-notificações ou vieses do tipo vício de amostragem ou de tendenciosidade por serem centros de referência e terem maior chance de receberem casos mais graves, se revela como valiosa maneira de conscientização, análise crítica, melhora da qualidade assistencial e dos resultados terapêuticos ${ }^{31,32}$.

Tabela 1 - Letalidade dos traumas cardiovasculares isolados.

\begin{tabular}{lcccc}
\hline Tipo de Lesão & Total de atendimentos & Sobreviventes & Óbitos & Letalidade \\
\hline Arterial & 89 & 47 & 42 & $47 \%$ \\
Venosa & 50 & 39 & 11 & $22 \%$ \\
Cardíaca & 11 & 2 & 9 & $81 \%$ \\
Total & 150 & 88 & 62 & $41 \%$ \\
\hline
\end{tabular}


O predomínio de homens jovens, dos fatores etiológicos, da propedêutica utilizada está de pleno acordo com os dados da literatura. Entretanto, chama a atenção à presença cada vez mais freqüente dos extremos das idades, conforme observado ${ }^{1,2,6-14,17,18,25,33-37}$. Não foram registrados acidentes com soldados das forças armadas na casuística apresentada. Por outro lado, uma significativa parcela das vítimas de agressão por arma de fogo foi representada por policiais militares e/ou civis e a grande similaridade dos dados deste estudo com as séries registradas a partir de conflitos armados, principalmente nos itens topografia anatômica, tipos de vasos lesados, manifestações clínicas à admissão, associação com outros órgãos ou sistemas e mortes hospitalares indica forte correlação no grande poder de destruição das armas de fogo utilizadas na violência urbana na qual se baseou esta grande série de $\operatorname{casos}^{2,10-14,17,27,38,39}$.

Constatou-se ainda que cerca de $1 / 4$ das lesões cardiovasculares tiveram como etiologia os traumas contusos, sendo na maioria das vezes devido a acidentes automobilísticos, não sendo registrada lesão cardíaca contusa. Sabe-se que $80 \%$ das vítimas com lesões cardíacas morrem no local do trauma/acidente. Os sobreviventes precisam ser rapidamente removidos para um centro hospitalar especializado em trauma, aonde se consegue taxa de sobrevida de até $75 \%$ nos pacientes que sofreram agressões por arma branca e de $35 \%$ naqueles com lesão produzida por arma de fogo $0^{36-40}$.

Corroborando achados por análise de regressão se observou que o local anatômico da lesão cardíaca e/ou a presença de tamponamento cardíaco não foram fatores isolados de mau prognóstico, mas sim agressão por arma de fogo, exsanguinação e necessidade de restauração ou manutenção da pressão arterial sistólica por drogas vasoativas ${ }^{38}$.

Atribui-se que grande parte das lesões vasculares graves, geralmente na aorta torácica e nas veias toraco-abdominais estão correlacionadas ao trauma contuso ${ }^{11,30,37,39,40}$, o que está parcialmente em concordância com os dados desde trabalho. Mas está em total desacordo a inexpressiva prevalência de lesões iatrogênicas observadas, já que dados internacionais encontraram prevalência de $22 \%$ até $40 \%{ }^{9}$, o que nos faz inferir como principais causas desta discordância a sub-notificação ou que as lesões, principalmente as iatrogênicas estejam sendo resolvidas nos próprios hospitais em que ocorreram.

À semelhança do que ocorreu nos conflitos armados com a introdução da evacuação rápida dos feridos nos campos de batalha, após a implantação do Serviço de Atendimento Médico de Emergência - SAMU nos grandes centros urbanos de nosso país aumentou a admissão de pacientes moribundos que antes não chegavam aos centros de trauma, o que nos obrigou a tomar medidas institucionais específicas $^{2,10,11,35,41}$. Apesar disso, a Escala de Trauma-Injúria de Órgãos da Associação Americana para a Cirurgia do Trauma (AAST-OIS) demonstrou correlação direta entre morte hospitalar e a intensidade do choque à admissão de vítimas de trauma cardíaco $^{38}$, resultado que condiz com a elevada taxa de letalidade cardíaca e chance de morte por lesão cardíaca na amostra avaliada.

A maior taxa de amputações secundárias em relação às amputações primárias deveu-se ao fato de que $10 \%$ das vítimas possuíam amputação traumática parcial passível com possibilidade de correção cirúrgica. Entretanto, temos observados uma incidência exponencialmente de lesões periféricas gravíssimas nos últimos dois anos, na maioria absoluta das vezes decorrentes dos acidentes com motocicletas ${ }^{19}$.

A análise da evolução hospitalar dos traumas cardiovasculares isolados visou minimizar possíveis fatores de confundimento. A diferença significativa encontrada entre os óbitos hospitalares devido a lesões arteriais e venosas isoladas e a maior chance de morte por lesões arteriais em relação às venosas isoladas reforça o modelo proposto. De forma geral tais lesões ocorrem devido às agressões penetrantes e/ou por traumas contusos que envolvem transferência de grande energia cinética em troncos vasculares desprovidos de tecido de sustentação capaz de conter a hemorragia e que está geralmente associada a outras lesões ${ }^{11,14,39,42}$, o que está de pleno acordo com os achados deste estudo. Das 11 mortes decorrentes das lesões venosas isoladas, oito (73\%) ocorreram no segmento das veias ilíaca-cava e o restante nas veias renal, femoral comum e subclávia (9\% cada).

Já as cinco lesões arteriais isoladas que evoluíram para óbito ocorreram nas femorais (33.6\%); ilíacas (29\%); subclávia (14.2\%); aorta (13.6\%) e carótidas (9.6\%). Estes dados coincidem com estudos que afirmam que as lesões dos vasos toraco-abdominais são responsáveis por altas taxas de morte no ambiente pré-hospitalar e também hospita$\operatorname{lar}^{1,2,11,39,41}$. De igual importância está o achado do predomínio de óbitos por lesões femorais e cervicais, sugerindo a necessidade de uma melhor qualificação daqueles envolvidos no atendimento pré-hospitalar, já que nesta casuística, significativa parcela destas vítimas foi atendida em choque hipovolêmico grave.

As principais complicações pós-operatórias descritas para as urgências e emergências cirúrgicas cardiovasculares são hemorragias com repercussões clínicas, complicações cárdio-pulmonares, infecções graves e síndrome de isquemia-reperfusão ${ }^{43-45}$, mas inúmeras lacunas científicas específicas sobre o tema ainda existem e fogem do objetivo deste trabalho.

Trata-se de uma das maiores séries mundial prospectiva sobre traumas cardiovasculares civis atendidos em um único centro. Os resultados demonstraram ser o homem jovem o mais acometido devido principalmente à violência interpessoal e aos acidentes automobilísticos. A grande maioria das lesões vasculares acometeu as extremidades e teve alta associação com lesões em outros órgãos ou sistemas. Foram identificados como fatores preditivos de mau prognóstico: choque hipovolêmico grave à admissão, lesão de grandes vasos arteriais e lesão cardíaca. Já a taxa de mortalidade global se correlacionou com o predomínio de lesões periféricas. 


\begin{abstract}
Background: To analyze the demographics and outcomes, from a large number of civilian cardiovascular (CCV) trauma cases, prospectively registered at a level I Brazilian Trauma Center. Methods: This paper deals with cardiovascular traumas studied in a prospective, descriptive and analytic way registered between 1998 through 2005. Results: Over 7-year period, 1000 patients were surgically approached. Most patients were young males with gunshot, stab wound/glasses and blunt trauma, distributed at the abdomen, neck, torso and more frequent at the limbs. The three main syndromes observed at admission were: hemorrhagic, ischemic and hemorrhagic/ischemic. Severe shock at admission was present in $34.6 \%$ of the patients, and non cardiovascular associated injuries were present in $85 \%$ of the cases. Most of the patients were operated on without preoperative tests. A second operation was required in 14\%, because of the following reasons: peripheral compartmental syndrome, acute arterial thrombosis or major hemorrhage. The amputation rate was $5.5 \%$ and mortality rate was $7.5 \%$ at discharge. Severe hemorrhagic shock and multiple organ failure were the main causes of death. Isolated CCV lesion was present in $15 \%$, with a global lethality of $41 \%$. Deaths were attributed to the following causes: venous (22\%), arterial (47\%) and cardiac (81\%) with statistical significance between cardiac lesions versus arterial plus venous lesions ( $p=0.01$; odds ratio $=7.37$ ) and arterial lesions versus venous lesions ( $p=0.01$; odds ratio 3.17). Conclusion: This large number of cases showed that the main victims were young men, involved on personal violence or vehicle crash accidents, with lesions more frequently found at the extremities with high association with non cardiovascular injuries. The negative prognostic predictive factors were severe shock, large artery lesions or cardiac injuries.
\end{abstract}

Key words: Cardiovascular System /lesions; Wounds and injuries; Ischemia; Reperfusion; Evidence-based medicine; Epidemiology; Vascular surgical procedures.

\section{REFERÊNCIAS}

1. Davis JH. History of trauma. In: Feliciano DV, Moore EE, Mattox KL. Trauma. $3^{\text {rd }}$ ed. Stamford: Appleton \& Lange; 1996. p. 3-13.

2. Rich MN. Historical and military aspects of vascular trauma (with lifetime reflections of Doctor Norman Rich). In: Rich NM, Mattox KL, Hirshberg A. Vascular trauma. $2^{\text {nd }}$ ed. Philadelphia: Elsevier Saunders; 2004. p. 3-72.

3. Peden M, McGee K, Sharma G. The injury chart book: a graphical overview of the global burden of injuries. Geneva: World Healthy Organization; 2002.

4. Peden M, McGee K, Krug E, editors. Injury: a leading cause of the global burden of disease, 2000. Geneva: World Healthy Organization; 2002.

5. Figueiredo LF, Cruz Jr RJ, Bruscagin V, Rasslan S, Silva MR. Iliac artery lesions as experimental models of vascular trauma inducing intraperitoneal and retroperitoneal hemorrhage. J Vasc Bras. 2002; 1(1):39-46.

6. Bez LG, Souza GS, Sena KA, Pinto DM, Simão-Filho C. Traumatismo vascular no membro superior por ponte extraanatômica: relato de caso. J Vasc Bras. 2003; 2(2):55-6; 141-4.

7. Silva AJM. Embolização de projétil de arma de fogo (PAF) provinda de veia poplítea para artéria pulmonar: acompanhamento e observação evolutiva durante 30 anos. J Vasc Bras. 2003; 2(2):14950; 164-6.

8. Medeiros CAF, Landim RM, Castro NA, Batista MAR, Kluge PD, Oliveira WC, Rocha EF, Luccas GC. Condutas no trauma penetrante da artéria axilar. J Vasc Bras. 2003; 2(3):225-8.

9. Shalabi R, Al Amri Y, Khoujah E. Vascular injuries of upper extremity. J Vasc Bras. 2006; 5(4):271-6.

10. Costa CA, Cabral PHO, Pinto PL, Pinheiro Jr. MJC, Santos NJV, Birolini D. Initial experience with hand replantation after traumatic amputation in a nonspecialized center. J Vasc Bras. 2006; 5(4):321-4.

11. Chiesa R, Moura MRL, Lucci C, Castellano R, Civilini F, Melissano G, Tshomba Y. Blunt trauma of the thoracic aorta: mechanisms involved, diagnosis and management. J Vasc Bras. 2003; 2(3):197-210.
12. Pinto DM, Bez LG, Costa-Val R, Campos-Christo SF, Miguel EV. Tratamento do trauma penetrante das artérias carótidas no hospital João XXIII. Comparação entre ligadura e reconstrução. J Vasc Bras. 2004; 3(3):247-52.

13. Sharma OP, Rawistscher R. Blunt vena azygos trauma: report of a case and review of world literature. J Trauma. 1999; 46(1):192-5.

14. Aun R. Ruptura traumática da aorta por traumatismo fechado (editorial). J Vasc Bras. 2007; 6(1):5-6.

15. Lovric Z, Wertheimer B, Candrlic K, Kuvezdic H, Lovric I, Medaric D, Janosi K. War injuries of major extremity vessels. J Trauma. 1994; 36(2):248-51.

16. Asfar S, Al-Ali J, Safar H, Al-Bader M, Farid E, Ali A, Kansou J. 155 vascular injuries: a retrospective study in Kuwait, 19922000. Eur J Surg. 2002; 168(11):626-30.

17. Goff J, Gillespie D, Rich N. Long-term follow-up of a superficial femoral vein injury: a case report from the vietnam vascular registry. J Trauma. 1998; 44(1):209-11.

18. Araújo GM, Mathias SB, Felipe Jr G. Dados epidemiológicos. In: Murilo R, Brito CJ, Vergara E, Meirelles S. Trauma vascular. Rio de Janeiro: Revinter; 2006. p. 74-82.

19. Campos-Christo SF, Miguel EV, Costa-Val R, Teixeira ELC. Amputação primária no trauma: indicações e aspectos legais. In: Murilo R, Brito CJ, Vergara E, Meirelles S. Trauma vascular. Rio de Janeiro: Revinter; 2006. p. 153-60.

20. Tanaka OU, Melo C. Reflexões sobre a avaliação em serviços de saúde e a adoção das abordagens qualitativa e quantitativa. In: Bosi MLM, Mercado FJ. Pesquisa qualitativa de serviços de saúde. Rio de Janeiro: Vozes. 2004; p.121-36.

21. Helman CG. Cultura, saúde e doença. In: Fatores culturais em epidemiologia. $4^{\mathrm{a}}$ ed. Porto Alegre: Artmed; 2003. p. 282-95.

22. Marasciulo ACE, Nassar SM. Noções sobre planejamento de estudos clínico-epidemiológicos. In: Drummond JP, Silva E, Coutinho M. Medicina baseada em evidências - novo paradigma assistencial e pedagógico. $2^{\mathrm{a}}$ ed. São Paulo: Atheneu; 2004. p.12540.

23. Duncan BB, Schmidt MI. Medicina baseada em evidências. In: Duncan BB, Schmidt MI, Giugliani ERJ. Medicina ambulatorial: condutas de atenção primária baseadas em evidências. $3^{\mathrm{a}}$ ed. Porto Alegre: Artmed; 2004. p. 31-40. 
24. Shaneyfelt T, Baum KD, Bell D, Teldstein D, Houston TK, Kaatz S, Whelan C, Green M. Instruments for evaluating education in evidence-based practice. A systematic review. JAMA. 2006; 296(9):1116-27.

25. Dib RPE. Como praticar a medicina baseada em evidências (editorial). J Vasc Bras. 2007; 6(1):1-4.

26. Yoshida WB. A nossa produção científica invisível (editorial). J Vasc Bras. 2005; 4(2):113.

27. Demetriades D, Murray J, Sinz B, Myles D, Chan L, Sathyaragiswaran L, Noguchi T, Bongard FS, Cryer GH, Gaspard DJ. Epidemiology of major trauma and trauma deaths in Los Angeles County. J Am Coll Surg. 1998; 187(4):373-83.

28. Sonneborn R, Andrade R, Bello F, Morales-Uribe CH, Razuk A, Soria A, et al. Vascular trauma in Latin America - a regional study. Surg Clin North Am. 2002; 82(1):182-94.

29. Morales-Uribe CH, Sanabria-Quiroga AE, Sierra-Jones JM. Vascular trauma in Colombia - experience of a level I trauma center in Medellin. Surg Clin North Am. 2002; 82(1):195-210.

30. Freedman EL, Safran MR, Meals RA. Automotive airbag-related with upper extremity injuries: a report of three cases. J Trauma. 1995; 38(4):577-81.

31. Hlaing T, Hollister L, Aaland M. Trauma registry data validation: essential for quality trauma care. J Trauma. 2006; 61(6):1400-7.

32. Guice K, Cassidy L, Mann NC. State trauma registries: survey and update-2004. J Trauma. 2007; 62(2):424-35.

33. Costa-Val R, Rios AV, Figueiredo Jr FA, Campos-Christo SF, Simão-Filho C, Campos-Christo C. Pseudo-aneurisma de artéria subclávia pós fratura de clavícula: relato de caso. Rev Bras Cir Cardiovasc. 1997; 12(4):384-6.

34. Costa-Val R, Rezende-Neto JB, Silva LPQ, Miguel EV, Souza TKDP. Trauma associado à variação anatômica dos vasos da base. J Vasc Bras. 2004; 3(1):55-56; 81-2.

35. Costa-Val R, Miguel EV, Simão Filho C. Onda vermelha - ações táticas que visam à abordagem de pacientes in extremis, no Hospital João XXIII, FHEMIG. Rev Angiol Cir Vasc. 2005; $5(2): 211-4$

36. Costa-Val R, Simão-Filho C, Campos-Christo SF, CamposChristo MB, Abrantes WL. Lesões traumáticas do coração: análise comparativa de dois grandes períodos numa grande população civil. An Paul Med Cir. 2000; 27(4):266-70.

37. Reis PEO, Chaves Filho M, Silva Jr CA. Via de acesso venoso no trauma. Rev Angiol Cir Vasc. 2003; 12(3):90-2.
38. Asensio JA, Berne JD, Demetriades D, Chan L, Murray J, Falabella A, Gomez H, Chahwan S, Velmahos G, Cornwell EE, Belzberg H, Shoemaker W, Berne T. One hundred five penetrating cardiac injuries: a 2-year prospective evaluation. J Trauma. 1998; 44(6):1073-82.

39. Robert A, Monahan T. Correlation of clinical characteristics and outcomes with injury scoring in blunt cardiac trauma. J Trauma. 2003; 54(3):509-15.

40. Moralez CH, Salinas CM, Henao CA, Patino PA, Munoz CM. Thoracoscopic pericardial window and penetrating cardiac trauma. J Traumam. 1997; 42(2):273-5.

41. Holcomb JB, Jenkins D, Rhee P, Johannignam J, Mahoney P, Mehta S, et al. Damage control resuscitation: directly addressing the early coagulopathy of trauma. J Trauma. 2007; 2(2):307-10.

42. Meyer DM, Jessen ME, Grayburn PA. Use of echocardiography to detect occult cardiac injury after penetrating thoracic trauma: a prospective study. J Trauma. 1995; 39(5):902-9.

43. Bonamigo TP, Becker M, Weber EL, Bianco C, Miranda F Jr, Figueiredo LF. Outcome after surgical repair of sealed rupture abdominal aortic aneurysms: a case-control study. Clinics. 2006; 61(1):29-34. Epub 2006 Mar 10.

44. Figueiredo LF, Cruz JR, Sannomiya P, Silva MR. Mechanisms of action of hypertonic saline resuscitation in severe sepsis and septic shock. Endocr Metab Immune Disord Drug Targets. 2006; 6(2):7-16

45. Rocha e Silva M, Poli de Figueiredo LF. Small volume hypertonic resuscitation of circulatory shock. Clinics. 2005; 60(2):159-72. Epub 2005 Apr 26.

Como citar este artigo:

Val RC, Campos-Christo SF, Simão Filho C, Bez LG, Marques MC, Miguel EV. Reflexões sobre o trauma cardiovascular civil a partir de um estudo prospectivo de 1000 casos atendidos em um centro de trauma de nível I. Rev Col Bras Cir. [periódico na Internet] 2008; 35(3). Disponível em URL: http://www.scielo.br/rcbc

Endereço para correspondência:

Ricardo Costa Val

Rua Boa Esperança, 341 / 101

Bairro Carmo-Sion.

30310-730 - Belo Horizonte - MG

Telefone: (31) 9972-60600.

E-mail: ricardocostaval@hotmail.com 